\title{
Dehydrogenated polycyclic aromatic hydrocarbons and UV bump (Research Note)
}

\author{
G. Malloci ${ }^{1}$, G. Mulas ${ }^{1}$, C. Cecchi-Pestellini ${ }^{1}$, and C. Joblin ${ }^{2}$ \\ ${ }^{1}$ Istituto Nazionale di Astrofisica - Osservatorio Astronomico di Cagliari, Strada n.54, Loc. Poggio dei Pini, 09012 Capoterra (CA), \\ Italy \\ e-mail: [gmalloci; gmulas; ccp]@ca.astro.it \\ 2 Centre d'Étude Spatiale des Rayonnements, Université de Toulouse, CNRS, Observatoire Midi-Pyrénées, \\ 9 avenue du Colonel Roche, 31028 Toulouse Cedex 04, France \\ e-mail: christine.joblin@cesr.fr
}

Received 12 May 2008 / Accepted 5 August 2008

\section{ABSTRACT}

\begin{abstract}
Context. Recent calculations have shown that the UV bump at about $217.5 \mathrm{~nm}$ in the extinction curve can be explained by a complex mixture of polycyclic aromatic hydrocarbons (PAHs) in several ionisation states. Other studies proposed that the carriers are a restricted population made of neutral and singly-ionised dehydrogenated coronene molecules $\left(\mathrm{C}_{24} \mathrm{H}_{n}, n \leq 3\right)$, in line with models of the hydrogenation state of interstellar PAHs predicting that medium-sized species are highly dehydrogenated.

Aims. To assess the observational consequences of the latter hypothesis we have undertaken a systematic theoretical study of the electronic spectra of dehydrogenated PAHs. We use our first results to see whether such spectra show strong general trends upon dehydrogenation.

Methods. We performed calculations using state-of-the-art techniques in the framework of the density functional theory (DFT) to obtain the electronic ground-state geometries, and of the time-dependent DFT to evaluate the electronic excited-state properties.

Results. We computed the absorption cross-section of the species $\mathrm{C}_{24} \mathrm{H}_{n}(n=12,10,8,6,4,2,0)$ in their neutral and cationic charge-states. Similar calculations were performed for other PAHs and their fully dehydrogenated counterparts.

Conclusions. $\pi$-electron energies are always found to be strongly affected by dehydrogenation. In all cases we examined, progressive dehydrogenation translates into a correspondingly progressive blue shift of the main electronic transitions. In particular, the $\pi \rightarrow \pi^{*}$ collective resonance becomes broader and bluer with dehydrogenation. Its calculated energy position is therefore predicted to fall in the gap between the UV bump and the far-UV rise of the extinction curve. Since this effect appears to be systematic, it poses a tight observational limit on the column density of strongly dehydrogenated medium-sized PAHs.
\end{abstract}

Key words. ISM: dust, extinction - ISM: lines and bands - ISM: molecules - ultraviolet: ISM - astrochemistry - molecular data

\section{Introduction}

First discovered by Stecher (1965), the absorption band centered at about $217.5 \mathrm{~nm}(\sim 5.7 \mathrm{eV})$ is the most intense interstellar extinction feature. With a relatively constant peak wavelength, the so-called "UV bump" has a Lorentz-like profile (e.g., Seaton 1979) whose width varies considerably from one line of sight to another (Fitzpatrick \& Massa 2007).

Both the strength and the position of the $217.5 \mathrm{~nm}$ feature suggest that it originates in some form of carbonaceous material, known to display strong $\pi \rightarrow \pi^{\star}$ electron transitions in this range. A large number of carrier candidates have been proposed over the years, amongst which size-restricted graphite particles (Stecher \& Donn 1965), fullerenes, $C_{60}$ in particular, (e.g., Braga et al. 1991; Iglesias-Groth 2004), polycyclic aromatic hydrocarbons (PAHs, Joblin 1992; Joblin et al. 1992), coallike material (Papoular et al. 1996), UV-processed hydrogenated amorphous carbon particles (Mennella et al. 1998), nano-sized hydrogenated carbon grains (Schnaiter et al. 1998), and carbon onions (Chhowalla et al. 2003).

Since each PAH displays strong $\pi \rightarrow \pi^{\star}$ transition in the $5.7 \mathrm{eV}$ region (Joblin 1992; Joblin et al. 1992; Draine 2003; Malloci et al. 2004), mixtures of PAHs must contribute to the $217.5 \mathrm{~nm}$ bump. Recently, Cecchi-Pestellini et al. (2008) showed that such mixtures can account for the entire bump, as well as its strength relative to the non-linear far-UV rise, depending on the distribution of charge states in the PAH mixtures. The UV bump was also attributed to a $\pi \rightarrow \pi^{\star}$ plasmon resonance from a very limited number of strongly dehydrogenated derivatives of coronene $\left(\mathrm{C}_{24} \mathrm{H}_{12}\right)$, namely $\mathrm{C}_{24} \mathrm{H}_{n}$ and $\mathrm{C}_{24} \mathrm{H}_{n}{ }^{+}$with $n \leq 3$. This assignment was made on the basis of DDA scattering calculations (Duley \& Seahra 1998), supported by laboratory studies of the UV absorption in amorphous carbon (Duley \& Lazarev 2004). The same molecules were also proposed as carriers of some of the diffuse interstellar bands (Duley 2006b). Indeed, models of the hydrogenation state of PAHs predict small and medium-sized PAHs ( $20-30$ carbon atoms) to be highly dehydrogenated (Le Page et al. 2003).

Since the effect of dehydrogenation on the electronic spectra of isolated PAHs is poorly characterized, we recently undertook a detailed study of the electronic excitation properties of dehydrogenated PAHs, using state-of-the-art quantum-chemical tools (Malloci et al. 2008). As a part of this more detailed work, we present in this paper our first results for the dehydrogenated derivatives of coronene $\left(\mathrm{C}_{24} \mathrm{H}_{12}\right)$, and completely dehydrogenated perylene $\left(\mathrm{C}_{20} \mathrm{H}_{10}\right)$ and ovalene $\left(\mathrm{C}_{32} \mathrm{H}_{14}\right)$. We use them to assess the specific case of the species introduced by Duley (2006a), whose computed spectral properties appear to be incompatible with the observed UV-bump, and search for general trends in the electron properties of dehydrogenated PAHs. 
Technical details of the calculations, as well as the justification for the choice of molecules considered, are given in Sect. 2. Results are presented and discussed in the astrophysical context in Sect. 3, and our conclusions are reported in Sect. 4.

\section{Methods}

\subsection{Computational details}

As in previous studies (Malloci et al. 2007a,b) we used density functional theory (DFT, Jones \& Gunnarsson 1989) for the electron ground state, and its time-dependent extension (TD-DFT, Marques \& Gross 2004) for electron excited states. Geometry optimisations were first performed using the computationally inexpensive $4-31 \mathrm{G}$ basis set, then refined with the $6-31+\mathrm{G}^{\star}$ basis (Frish et al. 1984). For this part of the work we used the hybrid B3LYP functional (Becke 1993), as implemented in the Gaussian-based DFT module of NWCHEM (Apra et al. 2005).

Starting from the geometry of each fully hydrogenated PAH (Malloci et al. 2007a), we considered its dehydrogenated derivatives. We considered only neutral and cationic charge-states, and found in all cases that the computed ground-states for neutrals and cations are the singlet and the doublet, respectively. All calculations were performed without symmetry constraints. To evaluate the effect of dehydrogenation on the electronic structure of the species considered, we performed exploratory HarthreeFock/4-31G calculations. Following Koopman's theorem (Eland 1984), we interpreted the one-electron molecular orbital energies thus obtained as vertical ionisation energies.

Keeping fixed the ground-state optimised geometries obtained above, we then computed the absorption cross-section $\sigma(E)$ using the real-time TD-DFT implementation of OCTOPUS (Marques et al. 2003), in the local-density approximation (Perdew \& Zunger 1981) as described in Malloci et al. (2007a). We previously showed (Malloci et al. 2004; Mulas et al. 2006) that the results of this method reproduce the overall far-UV behaviour of $\sigma(E)$, as seen in comparison with the experimental data available for a few neutral PAHs (Joblin 1992; Joblin et al. 1992). This includes the broad absorption peak dominated by $\sigma \rightarrow \sigma^{\star}$ transitions, which matches well both in position and width. The $\pi \rightarrow \pi^{\star}$ transitions from the visible to the UV range, while their absolute intensities are well reproduced, show energies that are systematically underestimated.

The TD-DFT formalism used in this work yields directly the time-dependent linear response of a given molecule after an impulsive perturbation, producing the whole spectrum in a single step (Marques et al. 2003). This differs from the most widely used frequency-space TD-DFT implementation, where the poles of the linear response function correspond to vertical excitation energies, and the pole strengths to the corresponding oscillator strengths (Casida 1995). We compare in Fig. 2 the experimental absorption spectra of neutral naphthalene (Gingell 1998), and anthracene (Joblin 1992; Joblin et al. 1992) with both the realtime TD-DFT (Malloci et al. 2004) and the frequency-space TDDFT (Malloci et al. 2007b) spectra. The latter results agree better with the laboratory data. However, computational costs scale steeply with the number of transitions and electronic excitations of molecules as large as those considered here are thus limited to the visible/near-UV part of the spectrum.

\subsection{Choice of molecules}

We studied the dehydrogenated derivatives of coronene $\mathrm{C}_{24} \mathrm{H}_{n}$ ( $n=10,8,6,4,2,0)$ in their neutral and cationic charge
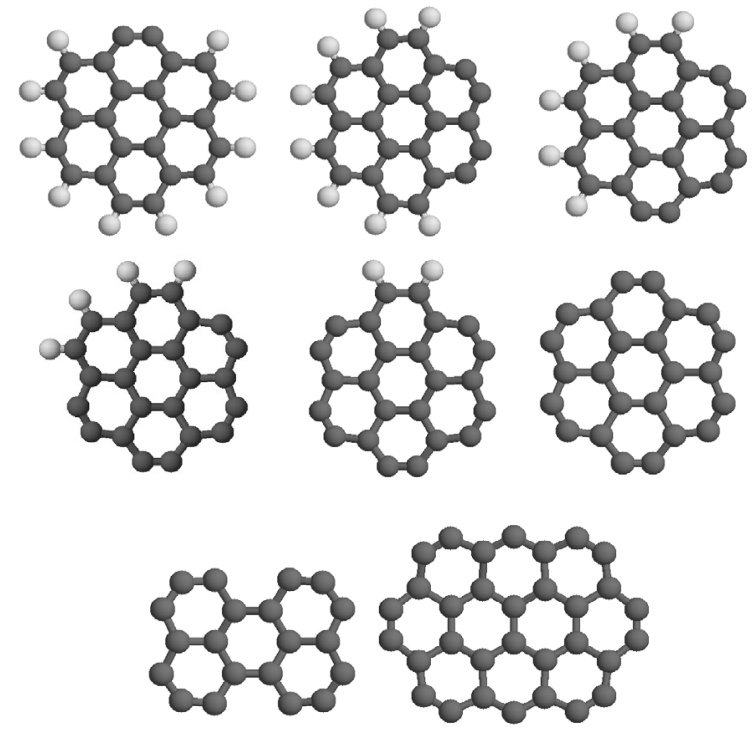

Fig. 1. Geometries of the dehydrogenated coronene molecules considered $\left(\mathrm{C}_{24} \mathrm{H}_{10}, \mathrm{C}_{24} \mathrm{H}_{8}, \mathrm{C}_{24} \mathrm{H}_{6}, \mathrm{C}_{24} \mathrm{H}_{4}, \mathrm{C}_{24} \mathrm{H}_{2}\right.$, and $\left.\mathrm{C}_{24}\right)$, and of the completely dehydrogenated perylene $\left(\mathrm{C}_{20}\right)$ and ovalene $\left(\mathrm{C}_{32}\right)$.

states. We included only species with an even number of hydrogen atoms, based on experimental mass spectra (Ekern et al. 1997) showing that dehydrogenation corresponding to loss of an even number of hydrogen atoms is dominant. In addition, experimental results on the dehydrogenation of the coronene cation (Joblin et al. 2008) show that, for $\mathrm{C}_{24} \mathrm{H}_{2 n}^{+}$molecules, only species containing adjacent paired hydrogens have to be considered. In particular, Joblin et al. (2008) found that the main hydrogen loss channel is the detachment of single atoms; however, the photodissociation rate for "lone" hydrogen atoms was observed to be much faster (about one order of magnitude) than for paired hydrogen atoms. This implies that dehydrogenated coronene derivatives containing adjacent paired hydrogens are more likely to be found in interstellar conditions. Moreover, it appears that the placement of the hydrogens at the periphery of the carbon skeleton has a minor effect on the main $\pi \rightarrow \pi^{\star}$ transitions. Such transitions depend mainly on the number of electrons populating the resonant $\pi$ and $\pi^{\star}$ molecular orbitals, which depend only on the number of peripheral hydrogen atoms and not on their specific position. As an example, Fig. 3 shows the comparison between the computed absorption cross-sections of two isomers of $\mathrm{C}_{24} \mathrm{H}_{10}{ }^{+}$, whose geometries are sketched in the same figure. As expected, with the exception of small details, the spectra of the two isomers are found to be almost indistinguishable in the energy range of astrophysical interest. Our study can therefore be restricted, without loss of generality for the scope of the present paper, to only the isomers of $\mathrm{C}_{24} \mathrm{H}_{n}$ $(n=10,8,6,4,2,0)$ shown in Fig. 1 . We additionally examined the completely dehydrogenated derivatives of perylene $\left(\mathrm{C}_{20} \mathrm{H}_{10}\right)$ and ovalene $\left(\mathrm{C}_{32} \mathrm{H}_{14}\right)$ cations, i.e. $\mathrm{C}_{20}^{+}$and $\mathrm{C}_{32}^{+}$, representative of non-symmetric, dehydrogenated, compact, medium-sized PAHs.

\section{Results and discussion}

\subsection{Dehydrogenation and electronic excitation properties}

According to Koopman's theorem the molecular orbital energies obtained at the Harthree-Fock / 4-31G level are interpreted as vertical ionisation energies. We report in Fig. 4 the energies corresponding to removal of an electron from both the highest 


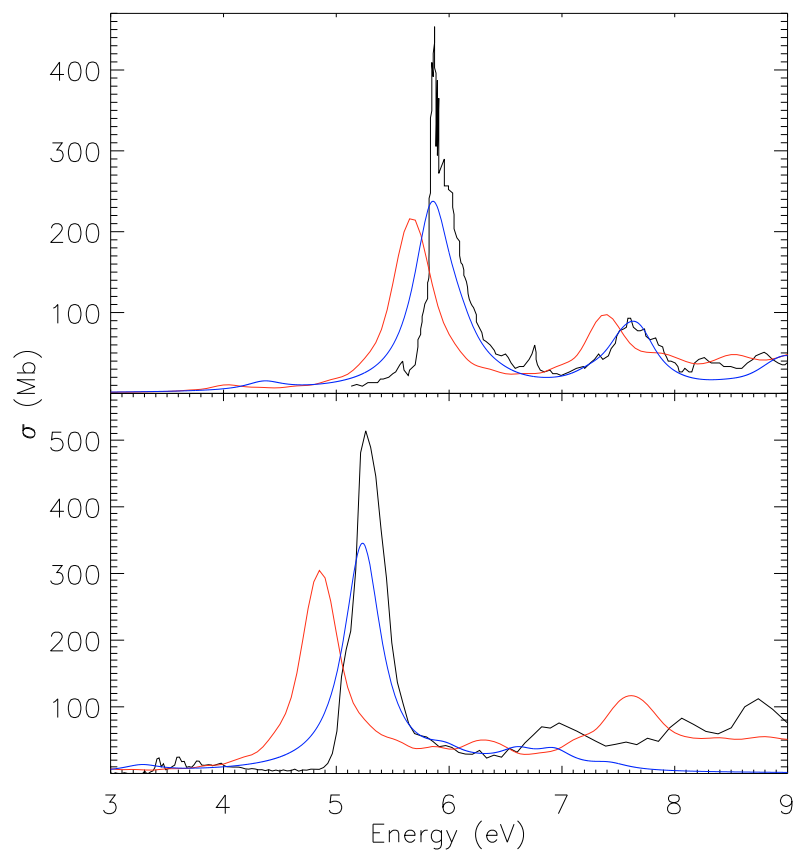

Fig. 2. Comparison between the experimental (black line) absorption cross-section $\sigma(E)$ in megabarns $\left(1 \mathrm{Mb}=10^{-18} \mathrm{~cm}^{2}\right)$ of neutral naphthalene (top, Gingell 1998), and anthracene (bottom, Joblin 1992; Joblin et al. 1992), and two different theoretical results: the real-time TD-DFT spectrum (red line, Malloci et al. 2004), and the frequency space TDDFT one (blue line, Malloci et al. 2007b).

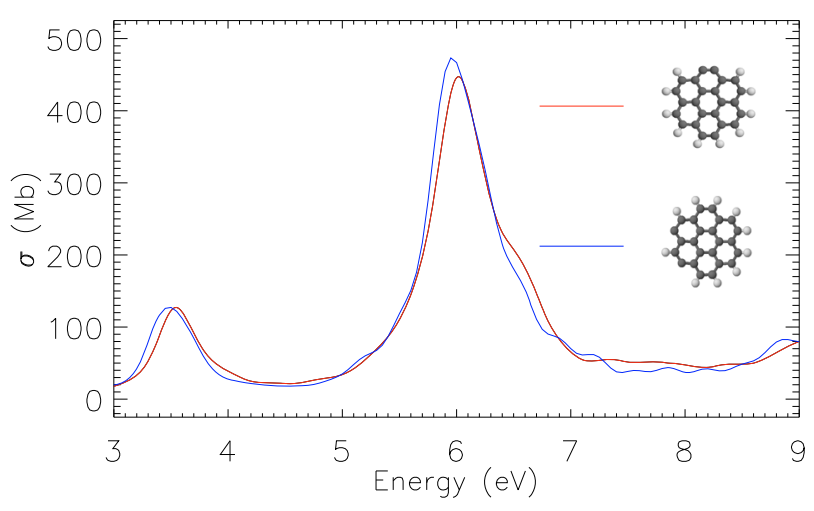

Fig. 3. Comparison between the computed absorption cross-section of the two inequivalent isomers of $\mathrm{C}_{24} \mathrm{H}_{10}{ }^{+}$sketched on the right.

occupied molecular orbital (IP1) and the molecular orbital just below it (IP2), as a function of the degree of hydrogenation $(x=0,2,4,6,8,10,12)$. In the model put forward by Duley (2006a) the low- and high-frequency cutoffs extracted from the interstellar extinction curve, at about 7.3 and $9.1 \mathrm{eV}$ respectively, are identified with the IP1 and IP2 of the carrier of the UV bump. These values were found to be in close agreement with those measured for neutral coronene, 7.29 and $9.13 \mathrm{eV}$, respectively (Clar et al. 1981). Based on the assumption that $\pi$-electron energies are little affected by dehydrogenation, this supported the proposal that a $\pi \rightarrow \pi^{*}$ plasmon resonance in neutral and singly-ionised dehydrogenated coronene molecules could generate the UV bump. However, we found an approximately constant change in ionization energy per hydrogen atom of about $0.1 \mathrm{eV}$ (cf. Fig. 4), suggesting that $\pi$-electron energies are indeed strongly affected by dehydrogenation. This is confirmed for the adiabatic first ionization energies computed via total energy

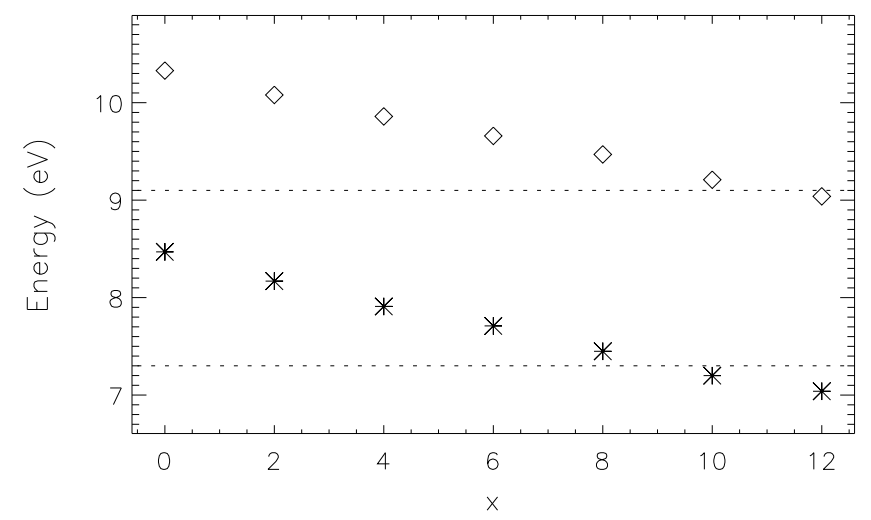

Fig. 4. First (asterisks) and second (diamonds) vertical ionisation energies of dehydrogenated coronene molecules $\mathrm{C}_{24} \mathrm{H}_{x}$, computed at the Harthree-Fock/4-31G level in the framework of Koopman's theorem, as a function of the degree of hydrogenation. The horizontal dotted lines correspond to the low- and high-frequency cutoffs extracted from the interstellar extinction curve in the model by Duley (2006a).

differences at the B3LYP/6-31+ $\mathrm{G}^{\star}$ level: 7.02, 7.23, 7.38, 7.61, $7.79,8.00$, and $8.25 \mathrm{eV}$, when going from $\mathrm{C}_{24} \mathrm{H}_{12}$ to $\mathrm{C}_{24}$.

The comparison between the computed absorption crosssections of the different dehydrogenated coronene molecules considered, in their neutral and cationic charge-states, is presented in Fig. 5. The corresponding comparisons between $\mathrm{C}_{20} \mathrm{H}_{10}^{+}$and $\mathrm{C}_{32} \mathrm{H}_{14}^{+}$and their fully dehydrogenated counterparts are shown in Fig. 6. For both neutral and singly-ionised coronene derivatives, progressive dehydrogenation translates into a correspondingly progressive blue shift of the main electronic transitions (Fig. 5). The $\pi \rightarrow \pi^{\star}$ collective resonance, in particular, becomes broader as well as bluer with dehydrogenation. The same holds true for the other PAHs reported in this work, namely perylene and ovalene (Fig. 6), suggesting that this is a general feature of the whole class of molecules.

\subsection{Astrophysical implications}

For a quantitative comparison with extinction, we estimate the expected spectral contrast of the $\pi \rightarrow \pi^{\star}$ collective resonance in a mixture of all the fully dehydrogenated species considered so far. The top panel of Fig. 7 shows the weighted sum of their absorption spectra, compared with the same weighted sum for their fully hydrogenated parent molecules. The $\pi \rightarrow \pi^{\star}$ feature appears to peak at $\sim 6.95 \mathrm{eV}(\sim 178 \mathrm{~nm})$, with an integrated intensity above the underlying continuum of about 1.3 times larger than the strong $\pi \rightarrow \pi^{\star}$ feature at $5.95 \mathrm{eV}$ associated with the hydrogenated counterparts.

Cecchi-Pestellini et al. (2008) showed that mixtures of a large number of fully hydrogenated PAHs can reproduce the UV bump and non-linear far-UV rise of the extinction curve. In particular, the UV bump is very accurately matched in position $(\sim 5.7 \mathrm{eV})$, intensity and width. Such a fit can only be obtained using mixtures including a much larger sample of PAHs than those included here. However, the position of the $\pi \rightarrow \pi^{\star}$ resonance, and its intensity per carbon atom, are relatively insensitive to the individual species considered. In all cases, its peak is well within the observed width of the UV bump. This is the reason why such transitions, in a mixture of hydrogenated PAHs, become blended, yielding a smooth feature which can be very similar to the observed UV bump profile.

In contrast, all completely dehydrogenated species considered here have the peak of their $\pi \rightarrow \pi^{\star}$ resonance about $\sim 1 \mathrm{eV}$ 


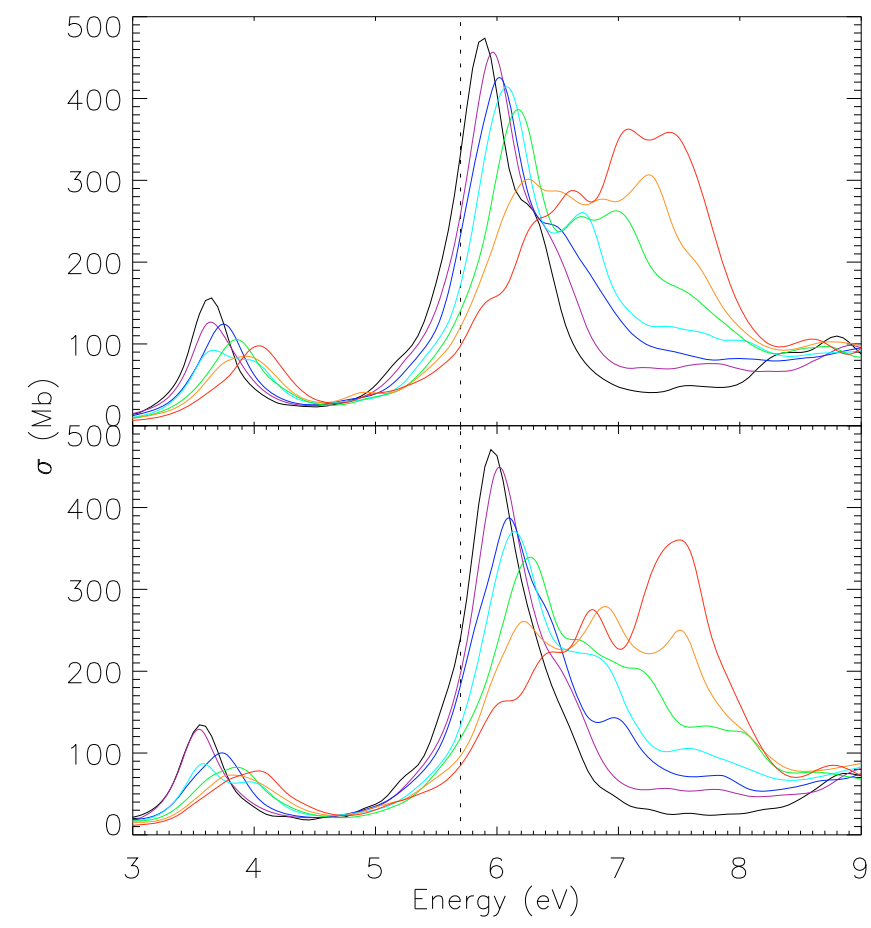

Fig. 5. Comparison between the computed absorption cross-sections of coronene $\mathrm{C}_{24} \mathrm{H}_{12}$ (black), $\mathrm{C}_{24} \mathrm{H}_{10}$ (violet), $\mathrm{C}_{24} \mathrm{H}_{8}$ (blue), $\mathrm{C}_{24} \mathrm{H}_{6}$ (cyan), $\mathrm{C}_{24} \mathrm{H}_{4}$ (green), $\mathrm{C}_{24} \mathrm{H}_{2}$ (orange), and $\mathrm{C}_{24}$ (red). Neutral molecules and their corresponding cations are displayed in the top and bottom panel, respectively. The dotted vertical line represents the position of the observed UV bump at $\sim 5.7 \mathrm{eV}$.

bluewards of the bump, in the gap between it and the onset of the non-linear far-UV rise. The position and intensity per carbon atom of the $\pi \rightarrow \pi^{\star}$ resonances of dehydrogenated PAHs, as a family, can be expected to be as insensitive to individual species as for their fully hydrogenated counterparts. A larger mixture, if this is the case, would yield a smoother feature than the one we calculated with the present sample, but generally with the same intensity and position.

Since no structure at $\simeq 7 \mathrm{eV}$ is detected in the average galactic interstellar extinction curve reported by Fitzpatrick \& Massa (2007), fully dehydrogenated PAHs must produce a peak smaller than the error of the interstellar extinction curve as illustrated in Fig. 7. Using a $2 \sigma$ upper limit, we can derive that the integrated intensity of the $\pi \rightarrow \pi^{\star}$ feature of dehydrogenated PAHs is less than $1 / 6$ of the bump area. Assuming that the bump is mainly due to hydrogenated PAHs (Cecchi-Pestellini et al. 2008) and using the factor given above for the relative integrated $\sigma(E)$, we derive that less than $1 / 8$ of the total abundance of carbon in PAHs is contained in strongly dehydrogenated PAHs. Assuming $N_{\mathrm{H}} \simeq 5.9 \times 10^{21} E_{B-V}$ (Whittet 2002), and applying the procedure of Cecchi-Pestellini et al. (2008) to the average extinction curve $\left(R_{V} \simeq 3.01\right.$ from Fitzpatrick \& Massa 2007) yields a value of $\sim 150 \mathrm{ppM}$ for the abundance of $\mathrm{C}$ locked in PAHs (including both the free-flying ones and those clustered in very small grains); the upper limit of the contribution of dehydrogenated PAHs to the extinction curve can then be translated into an upper limit of $\sim 18 \mathrm{ppM}$ for the carbon atoms contained in them. This numeric result is obviously dependent on the choice to compare against the average interstellar extinction curve, and would be different for a different choice. We showed in Cecchi-Pestellini et al. (2008) that the fraction of carbon contained in free-flying

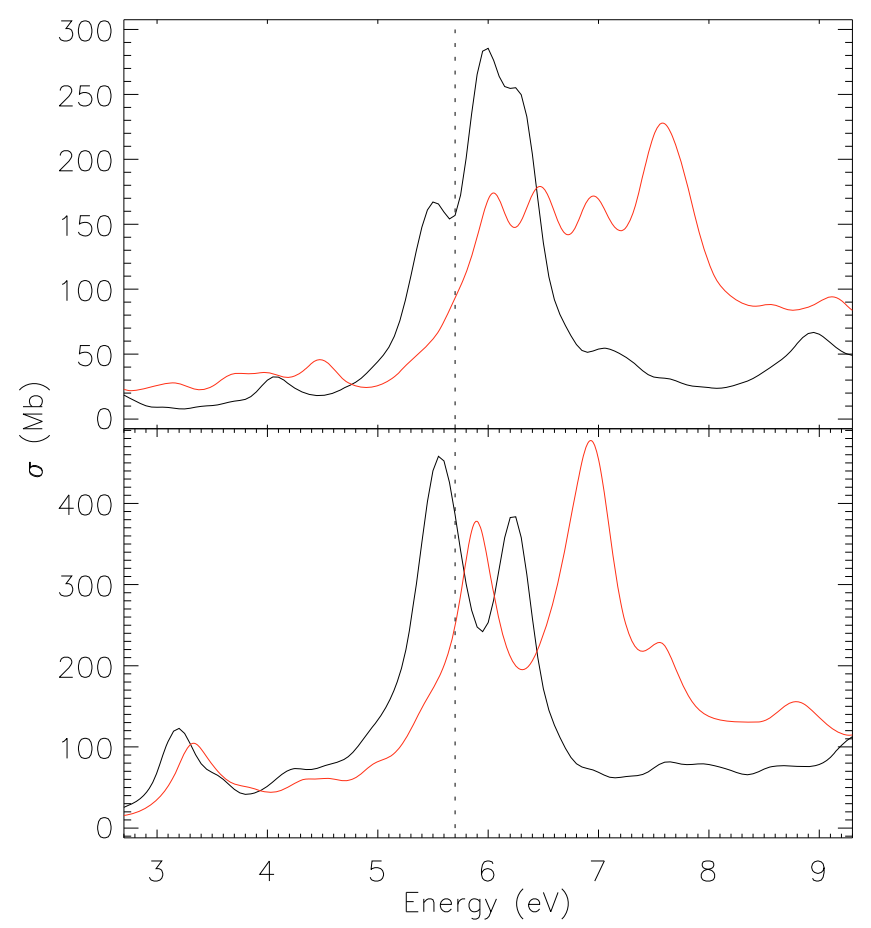

Fig. 6. Comparison between the computed absorption cross-sections of fully hydrogenated (black), and fully dehydrogenated (red) perylene cation $\left(\mathrm{C}_{20} \mathrm{H}_{10}^{+}\right)$(top panel) and ovalene cation $\left(\mathrm{C}_{32} \mathrm{H}_{14}^{+}\right)$(bottom panel).

and clustered PAHs is quite variable, from 85 to $187 \mathrm{ppm}$ in a sample of lines of sight.

Our upper limit can be compared with those found by Clayton et al. (2003) regarding the column density of specific small, neutral, fully hydrogenated PAHs in specific lines of sight, based on laboratory spectra and Hubble Space Telescope observations. Their results are more stringent thanks to the stronger spectral contrast of the absorption bands in their experimental spectra, coupled with the matching high resolving power of the STIS instrument. For the present comparison, however, such higher resolving power would not help, given the broader nature of the spectral signature being sought.

\section{Conclusions}

PAHs are expected to exist in a wide variety of interstellar environments, in a complex statistical equilibrium of different charge and hydrogenation states (see e.g., Tielens 2005). Modelling studies suggest that intermediate-size PAHs in the range of 20-30 carbon atoms are stripped of most of their hydrogen atoms but still survive under the conditions of the diffuse interstellar medium (Le Page et al. 2003).

We undertook a systematic theoretical study of the effects of dehydrogenation on the electron properties of neutral and ionised PAHs using state-of-the-art quantum-chemical techniques in the framework of the real-time TD-DFT. For all of the species considered so far, we found that, regardless of chargestate and molecular size, the $\pi \rightarrow \pi^{\star}$ collective resonance broadens and shifts to higher energies with increasing dehydrogenation.

When the molecule is half dehydrogenated or more, the band is shifted enough to fall between the bump and the far-UV rise of the extinction curve. The associated species cannot be the carriers of the bump, contrary to the proposal of Duley (2006a). 


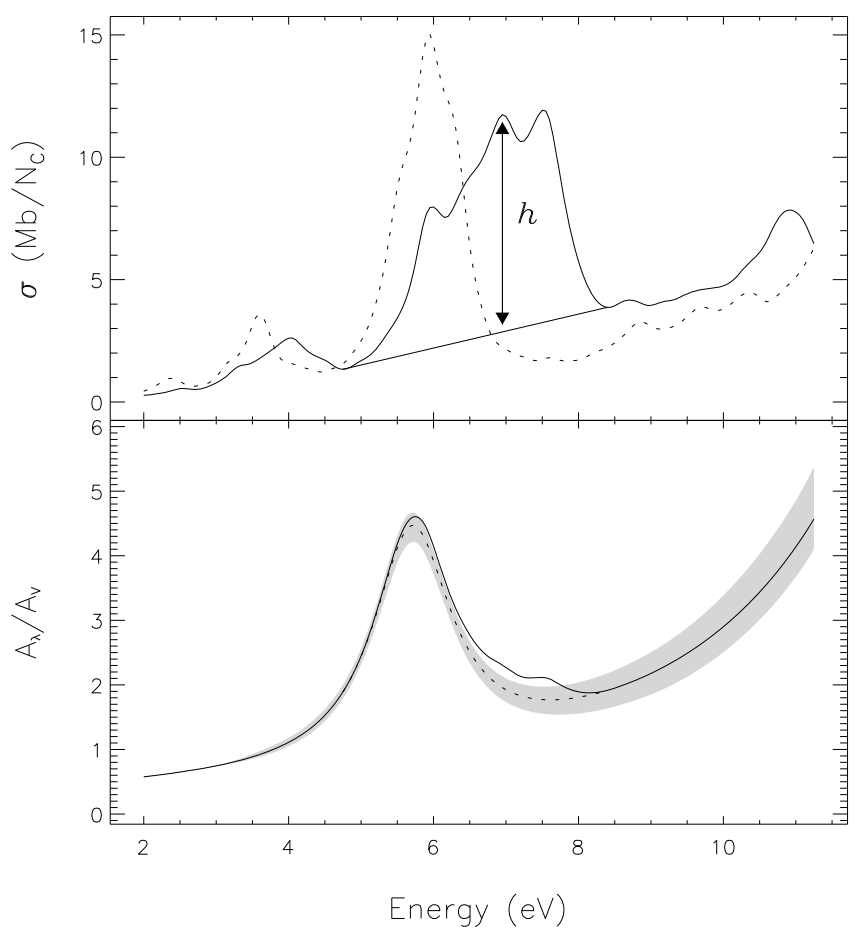

Fig. 7. Top panel: weighted sum of the computed absorption crosssections of $\mathrm{C}_{20}^{+}, \mathrm{C}_{24}, \mathrm{C}_{24}^{+}$, and $\mathrm{C}_{32}^{+}$(continuous line), and $\mathrm{C}_{20} \mathrm{H}_{10}^{+}, \mathrm{C}_{24} \mathrm{H}_{12}$, $\mathrm{C}_{24} \mathrm{H}_{12}^{+}$, and $\mathrm{C}_{32} \mathrm{H}_{14}^{+}$(dotted line), using as weight the inverse of the number of $\mathrm{C}$ atoms in each molecule. Bottom panel: comparison between the average extinction curve (dotted line, Fitzpatrick \& Massa 2007), with the $1 \sigma$ region in gray shade, and the average extinction curve + the amount of dehydrogenated PAHs which would make the $\pi \rightarrow \pi^{\star}$ feature detectable at $2 \sigma$ (continuous line).

While it is overreaching to draw definitive conclusions based on the few cases considered, our upper limits are due to systematic effects, and are thus unlikely to depend strongly on the specific sample of molecules considered. Nonetheless, a larger study, which is underway (Malloci et al. 2008), will be needed to assess to what extent the effect of dehydrogenation that we observed in UV spectra of PAHs is systematic for the whole class.

Acknowledgements. G. Malloci acknowledges financial support by Regione Autonoma della Sardegna. G. M., G. M., and C. C.-P. acknowledge financial support by MIUR under project CyberSar, call 1575/2004 of PON 2000-2006. We acknowledge the authors of OcTOPUS and the authors of NWCHEM, A Computational Chemistry Package for Parallel Computers, Version 4.7 (2005),
PNNL, Richland, Washington, USA. Parts of these simulations were carried out at CINECA (Bologna).

\section{References}

Apra, E., Windus, T. L., Straatsma, T. P., et al. 2005, NWChem, A Computational Chemistry Package for Parallel Computers, Version 4.7

Becke, A. D. 1993, J. Chem. Phys., 98, 5648

Braga, M., Larsson, S., Rosen, A., \& Volosov, A. 1991, A\&A, 245, 232

Casida, M. E. 1995, in Recent Advances in Density Functional Theory, Vol. I, ed. D. P. Chong (Singapore: World Scientific)

Cecchi-Pestellini, C., Malloci, G., Mulas, G., Joblin, C., \& Williams, D. A. 2008, A\&A, 486, L25

Chhowalla, M., Wang, H., Sano, N., et al. 2003, Phys. Rev. Lett., 90, 155504

Clar, E., Robertson, J. M., Schlögl, R., \& Schmidt, W. 1981, J. Am. Chem. Soc., 103,1320

Clayton, G. C., Gordon, K. D., Salama, F., et al. 2003, ApJ, 592, 947

Draine, B. T. 2003, ARA\&A, 41, 241

Duley, W. W. 2006a, ApJ, 639, L59

Duley, W. W. 2006b, ApJ, 643, L21

Duley, W. W., \& Lazarev, S. 2004, ApJ, 612, L33

Duley, W. W., \& Seahra, S. 1998, ApJ, 507, 874

Ekern, S. P., Marshall, A. G., Szczepanski, J., \& Vala, M. 1997, ApJ, 488, L39

Eland, J. H. D. 1984, Photoelectron Spectroscopy (London: Butterworths)

Fitzpatrick, E. L., \& Massa, D. 2007, ApJ, 663, 320

Frish, M. J., Pople, J. A., \& Binkley, J. S. 1984, J. Chem. Phys., 80, 3265

Gingell, J. M. 1998, Faraday Discussion, 109, 400

Iglesias-Groth, S. 2004, ApJ, 608, L37

Joblin, C. 1992, Ph.D. Thesis, Université Paris 7

Joblin, C., Léger, A., \& Martin, P. 1992, ApJ, 393, L79

Joblin, C., Boissel, P., Armengaud, M., \& Frabel, P. 2008, in preparation

Jones, R. O., \& Gunnarsson, O. 1989, Rev. Modern Phys., 61, 689

Le Page, V., Snow, T. P., \& Bierbaum, V. M. 2003, ApJ, 584, 316

Malloci, G., Mulas, G., \& Joblin, C. 2004, A\&A, 426, 105

Malloci, G., Joblin, C., \& Mulas, G. 2007a, A\&A, 462, 627

Malloci, G., Mulas, G., Cappellini, G., \& Joblin, C. 2007b, Chem. Phys., 340, 43

Malloci, G., Mulas, G., Cecchi-Pestellini, C., \& Joblin, C. 2008, in preparation

Marques, M. A. L., Castro, A., Bertsch, G. F., \& Rubio, A. 2003, Comput. Phys. Commun., 151, 60

Marques, M. A. L., \& Gross, E. K. U. 2004, Ann. Rev. Phys. Chem., 55, 3425

Mennella, V., Colangeli, L., Bussoletti, E., Palumbo, P., \& Rotundi, A. 1998, ApJ, 507, L177

Mulas, G., Malloci, G., Joblin, C., \& Toublanc, D. 2006, A\&A, 460, 93

Papoular, R., Conard, J., Guillois, O., et al. 1996, A\&A, 315, 222

Perdew, J. P., \& Zunger, A. 1981, Phys. Rev. B, 23, 5048

Schnaiter, M., Mutschke, H., Dorschner, J., Henning, T., \& Salama, F. 1998, ApJ, 498, 486

Seaton, M. J. 1979, MNRAS, 187, 73P

Stecher, T. P. 1965, ApJ, 142, 1683

Stecher, T. P., \& Donn, B. 1965, ApJ, 142, 1681

Tielens, A. G. G. M. 2005, The Physics and Chemistry of the Interstellar Medium (Cambridge: Cambridge University Press)

Whittet, D. C. B. 2002, Dust in the galactic environment 2nd ed. (The Graduate Series in Astronomy, Bristol: Institute of Physics (IOP) Publishing, 1992) 